\title{
Informes
}

\section{TALLER SOBRE "LA PARTICIPACIÓN SOCIAL EN LA RECONSTRUCCIÓN, CON PARTICULAR REFERENCIA AL PAPEL. DE LA MUJER"}

El 5, 6 y 7 de noviembre pasado se llevó a cabo en El Colegio de México el taller dedicado a analizar "la participación de la mujer en la reconstrucción", auspiciado por el Programa Interdisciplinario de la Mujer (PIEM) de El Colegio y UNICEF.

\section{Antecedentes que guiaron la organización del taller}

Para los habitantes de las vecindades, el terremoto significó no sólo la pérdida de su vivienda (además, en muchas ocasiones, de familiares y empleos), sino también la necesidad de participar en nuevas experiencias de movilización y organización vecinal, exigidas por los programas de reconstrucción.

En la mayoría de las vecindades esta necesidad de participación y organización es una experiencia colectiva inédita, sin antecedentes más allá de las tradicionales formas de sobrevivencia y de cooperación vecinal para el mantenimiento y arreglo del inmueble, para las fiestas y acontecimientos de la vida cotidiana. La organización y lucha inquilinaria de los vecinos del centro de la ciudad tiene antecedentes y desarrollo sólo en algunos barrios y colonias: Tepito (Movimiento Cultural Tepito Arte Acá y el N̄ero en la Cultura), Guerrero (Unión de Vecinos de la colonia Guerrero) y Unión Popular de Inquilinos de la colonia Morelos y Peña Morelos). En estos casos, como en todas las organizaciones populares de base territorial, las mujeres constituyen el soporte principal de las acciones colectivas que se realizan para mejorar las condiciones de vida urbana y defender los derechos ciudadanos.

Múltiples experiencias demuestran que las mujeres se convierten en los sujetos sociales más activos y permanentes de los movimientos urbanos y organizaciones vecinales, no obstante las dificultades y limitaciones que deben enfrentar para incorporarse a la participación pública. El papel y problemática específica de la mujer en la sociedad determina ese conjunto de dificultades y limitaciones, pero también de allí provienen sus potencialidades y capacidades para el trabajo y organización comunitaria.

Para la gran mayoría de las mujeres que han sufrido la pérdida de la vivienda a causa de los sismos, la participación en la construcción y ges- 
tión social de la nueva vivienda implica iniciar un proceso de aprendizaje individual y colectivo que debe estimularse atendiendo a sus problemas, condiciones y antecedentes sociales y urbanos concretos. Esto con la perspectiva de desarrollar un nuevo proceso social de gestión y participación vecinal, en el que las mujeres puedan constituirse en protagonistas fundamentales.

\section{Objetivos y desarrollo del taller}

Frente a la problemática enunciada, se propuso la realización de varias reuniones de trabajo con personas activamente involucradas en los diferentes tipos de instituciones y organizaciones que están participando en la reconstrucción de las zonas afectadas por los sismos. Ellas incluirían instituciones oficiales que a diferentes niveles han desarrollado proyectos de reconstrucción, organizaciones vecinales que están promoviendo y participando en la reconstrucción de sus barrios, y organismos universitarios y técnicos no gubernamentales que apoyan, a través de estudios, proyectos y acciones, la actividad de esas organizaciones populares.

El objetivo de esas reuniones de trabajo era presentar y discutir:

a) Algunos elementos conceptuales referidos a la organización popular, a la participación de la mujer en la misma, particularmente en programas barriales de reconstrucción y mejoramiento del hábitat.

b) Experiencias concretas de participación de la mujer en algunos programas específicos de reconstrucción, siguiendo una pauta de exposición previamente fijada, que permitiera establecer comparaciones, extraer conclusiones y realizar un balance del conjunto de casos analizados.

El taller se desarrolló en tres sesiones de cuatro horas cada una. En la primera de ellas se comenzó con una introducción por parte de las coordinadoras, quienes hicieron una breve exposición de la temática más amplia de la vivienda para los sectores pobres de la ciudad de México y del impacto de los sismos sobre el sector habitacional, aportando asimismo elementos teóricos sobre la participación de la mujer en los problemas urbanos. Luego se pasó a presentar y discutir los análisis de caso, que ocuparon el resto de las sesiones. Estas presentaciones fueron realizadas básicamente por representantes de la Regional de Mujeres del Valle de México (Conamup), de la Unión Popular Nueva Tenochtitlán, de la Unión de Vecinos de la colonia Doctores, de la Unión Popular de Inquilinos de la colonia Morelos (y Peña Morelos), de los Campamentos Unidos de la colonia Guerrero, del grupo de las Trece Vecindades de Tepito, de la cooperativa Superación de la Vivienda de la colonia Morelos y de la Unión de Solicitantes de Vivienda La Libertad; todas organizaciones populares independientes que ya existían antes de los sismos, o que surgieron a partir de ese momento. 
En varios de los casos presentados participaron además de las citadas organizaciones, miembros de equipos técnicos de apoyo, ya sea correspondientes a las universidades como a grupos de profesionales independientes (UAM-Azcapotzalco; Casa y Ciudad; Copevi; Instituto de Capacitación y Educación Popular; Cosvi; PDF y Procali (para Ciudad Guzmán).

Sólo en el caso del Plan Bondojito, correspondiente a la delegación Gustavo A. Madero del Distrito Federal, se trató de una presentación del sector oficial.

Los análisis de casos se acompañaron de presentaciones de audiovisuales, diapositivas, etc., lo cual permitió tener un contacto mucho más vivo con las diferentes experiencias. Algunos de estos audiovisules fueron realizados por las mismas organizaciones.

Las sesiones contaron con una nutrida concurrencia, sobre todo de mujeres pertenecientes a las diferentes organizaciones participantes; ellas tomaron parte activamente en las discusiones que se generaron, lo cual dio la pauta del interés existente en el tema tratado, así como de la falta de otras instancias para la discusión conjunta de los problemas de las mujeres de las diferentes colonias afectadas por los sismos. A pedido de las propias participantes se acordó realizar una sesión extra, en la cual se pudieran elaborar conclusiones de todo el material presentado y de las discusiones generadas, así como también esbozar algunas estrategias para la acción futura.

\section{Conclusiones y recomendaciones del taller}

a) A través de las exposiciones, las mujeres reconocieron que los sismos habían generado una situación de cambio muy importante en sus vidas. De distintas maneras expresaron que al haber sufrido la pérdida de sus viviendas, al haberse caído los muros, descubrieron el ámbito que está más allá de sus hogares y se vieron impulsadas a participar dentro de la comunidad, para resolver los problemas más urgentes que fueron apareciendo y luego para reconstruir sus habitaciones y su barrio.

Como los hombres deben salir a trabajar, las mujeres fueron las que tuvieron que enfrentar esos problemas, y para ello debieron aprender muchas cosas nuevas que por cierto las han enriquecido. Así, admitieron que esas experiencias nuevas las han cambiado ("esta cuestión del feminismo no nos viene de los libros, por la teoría, sino de la práctica cotidiana"), las han ayudado a capacitarse para desarrollar nuevas actividades, así como a revalorizar su trabajo. Por ello plantean que cuando se acabe la reconstrucción ya no quieren volver a sus casas y olvidar lo aprendido, sobre todo ya no quieren que el hombre "las mande", porque se han dado cuenta de su papel preponderante en todas las iniciativas plan- 
teadas y acciones desarrolladas a partir de los sismos. Ello les ha permitido tomar conciencia de sus valores y posibilidades.

b) Con respecto al tema de la organización de las mujeres para seguir actuando, la gran mayoría estuvo de acuerdo en que resulta necesario tener sus propias instancias organizativas, para defenderse y discutir sus problemas comunes ("debemos tratar de unirnos y esa unión es para formar una fuerza para que se nos respete"). Esas instancias deberán adecuarse en las condiciones de cada lugar y organización vecinal existente.

También se reconoció que, si bien las mujeres deben organizarse, se debe tener cuidado de "no armar otra lucha"; esta debe ser conciliable con las otras luchas de la comunidad.

Algunas propusieron organizar eventos de las mujeres dentro de la Coordinadora Única de Damnificados, pero esta propuesta no es expresión de las inquietudes de todas las participantes del taller; en este sentido es necesario aclarar que algunas organizaciones dijeron que "no hacen política" mientras que otras se encuentran integradas a esa Coordinadora, actuando en la reconstrucción con una perspectiva política bastante clara.

c) En cuanto a la reconstrucción de las viviendas, las mujeres han colaborado muy activamente en las tareas involucradas en la misma. En algunas colonias, ellas participan en la construcción misma (dos a tres horas por día en la colonia Doctores) y en otros casos han participado en el proyecto (planteando sus necesidades y puntos de vista), en la supervisión y control de las obras, enfrentándose a veces a las autoridades.

En general las mujeres expresaron que no sienten que trabajar en la autoconstrucción de sus viviendas constituya una forma de explotación, porque aunque tengan que aportar un mayor trabajo, ello les permitirá, por otro lado, obtener una casa a precios menores, aprender un oficio, y sumar nuevas experiencias a su vida cotidiana. Además, el proceso de autoconstrucción permite agregar otras formas de unión de los vecinos, para fortalecer sus organizaciones.

También señalaron que la experiencia de organizaciones independientes como (Usovi) Unión de Solicitantes de Vivienda sirvió a las mujeres para coordinar y reglamentar más eficientemente su colaboración en las distintas actividades y responsabilidades dentro del trabajo colectivo.

Las ponencias presentadas, así como las discusiones recogidas y las conclusiones y recomendaciones del taller (que aquí sólo hemos transcrito de manera muy resumida) aparecerán publicadas en un libro promovido y apoyado por las instituciones patrocinantes (PIEM y UNICEF). 


\section{SEMINARIO CIUDAD DE MÉXICO: ECONOMÍA, URBANISMO, ORGANIZACIÓN POLÍTICA, IMPACTO DE LOS SISMOS, PLANEACIÓN Y PERSPECTIVAS FUTURAS}

Entre el 21 y 24 de octubre de 1986 se realizó en El Colegio de México el seminario "Ciudad de México: economía, urbanismo, organización política, impacto de los sismos, planeación y perspectivas futuras" patrocinado por El Colegio y el Departamento del Distrito Federal.

En la reunión participaron como ponentes investigadores de El Colegio y de otras instituciones de enseñanza superior, así como funcionarios del Departamento del Distrito Federal.

A continuación se transcribe el discurso inaugural que el doctor Gustavo Garza presentó en ese evento y que explica las razones de haber incluido los siete temas que conformaron el seminario.

La ciudad de México se ha transformado durante el siglo XX en una de las más grandes metrópolis del planeta constituyéndose, indefectiblemente, en un fenómeno histórico de gran interés para la teoría y la práctica de la planificación metropolitana.

Para la economía del país la ciudad de México es de suma importancia, pues produce alrededor de la mitad de sus actividades industriales, comerciales y de servicios, por lo que absorbe cerca de $45 \%$ del producto interno bruto nacional.

A cierto nivel de generalidad, se puede decir que la dinámica de sus principales sectores económicos determinan su crecimiento demográfico y urbanístico y la compleja problemática que el proceso global conlleva. Esta concepción parte de la tesis que considera al proceso de industrialización como la génesis de la expansión económica, demográfica y urbanística. Así, el crecimiento industrial implica un crecimiento comercial, de servicios, transporte, construcción, etc., adicional al preexistente. Considerando que no se dispone de ninguna investigación exhaustiva sobre este conjunto de características económicas básicas de la capital del país, en la primera parte de este evento titulada "Crecimiento económico de la ciudad de México durante el siglo XX", se presentan algunos estudios monográficos sobre la importancia económica de la ciudad de México según sectores principales. Sería sumamente importante que estos trabajos iniciaran la investigación rigurosa de la base económica de la urbe para estar en posibilidades de planear su crecimiento futuro.

La población se traslada hacia donde el capital crea empleos. De esta suerte, como corolario del crecimiento económico de la ciudad de México, se produce su explosiva dinámica demográfica. En 1900 la ciudad tenía 344 mil habitantes que aumentaron a 661 mil en 1921. En 1930 su área urbana rebasaba el millón de habitantes (1 millón 48 
mil) y alcanzó 1.5 millones en 1940, que casi se duplicaron en 1950, cuando residían en ella 2.8 millones de personas. Para 1960 tenía 4.9 millones, que aumentaron sistemáticamente a 8.6 millones en 1970, a 13.8 millones en 1980, y se estima, a 17.2 millones en 1985 . La expansión de la mancha urbana ocurre simultáneamente con el crecimiento económico y demográfico. En lo que va del siglo, el desarrollo urbano de la ciudad de México se puede dividir en cuatro etapas: la primera va de 1900 a 1930 , cuando el centro de la ciudad era más dinámico que las delegaciones que lo rodeaban dentro de un primer contorno; la segunda corresponde al periodo 1930-1950, caracterizado por un crecimiento más elevado que el de la etapa anterior y una mayor dinámica en las delegaciones periféricas a los antiguos doce cuarteles; la tercera, o etapa de metropolización, se inicia en 1950, cuando la ciudad rebasa los límites del Distrito Federal extendiéndose hacia el Estado de México y termina en 1980; la actual cuarta etapa se inicia en los años ochenta, cuando la zona metropolitana se traslapa con la adyacente de Toluca iniciando su expansión megalopolitana.

El estudio de este proceso de expansión demográfico-geográfica de la ciudad de México se aborda en la segunda parte de este acto denominada, "El proceso de metropolización de la ciudad de México, 19001980", en la cual un conjunto de especialistas analiza las peculiaridades de la expansión física, demográfica y urbanística de la capital de la República. Aunque este tipo de estudios están más desarrollados que los económicos, sería muy aconsejable iniciar investigaciones con el rigor científico necesario para intentar interpretar la expansión metropolitana según el conjunto de teorías que tratan de explicar la estructuración del espacio intraurbano.

El tejido urbano de la ciudad de México se ha ido expandiendo en forma extraordinaria. De tener una extensión de $86 \mathrm{~km}^{2}$ en 1930 , aumentó a $117 \mathrm{Km}^{2}$ en 1940; a $240 \mathrm{~km}^{2}$ en 1935, y a $650 \mathrm{~km}^{2}$ en 1970 , alcanzando en la actualidad alrededor de $1200 \mathrm{~km}^{2}$. Para hacer posible este crecimiento físico, ha sido necesaria la construcción de un colosal conjunto de obras de infraestructura y servicios urbanos que hacen de la ciudad de México la más extensa, compleja, importante y prolongada obra que la nación mexicana ha producido en todo su desarrollo histórico, con obras viales, metro, drenaje profundo, sistema elećtrico, abastecimiento de agua, sistema de hidrocarburos, comunicaciones y transportes, sistema educativo y hospitalario, etc. La presentación esquemática de este conjunto de obras y servicios está comprendida en la tercera parte de este seminario, denominada "Infraestructura y servicios públicos en la ciudad de México". La capital de la nación constituye su espacio político más importante. En primer lugar, por ser el asiento de los poderes de la federación 
y por siglos el centro político nacional. En segundo lugar, porque sus 17 millones de habitantes constituyen la mayor aglomeración de ciudadanos en México, lo que presenta una problemática especial en relación a su representación política y participación ciudadana. La dimensión política de la ciudad de México es materia de la cuarta parte, titulada "Gobierno y organización política", en ella se presentan estos aspectos, que son objeto actualmente de un profundo debate nacional. El 19 de septiembre de 1985 la ciudad de México, cuya fisonomía económica, demográfica, urbanística y política se acaba de mencionar, sufrió un sismo con muy graves consecuencias en vidas, edificaciones y servicios públicos. Las causas, consecuencias y acciones emprendidas para enfrentar este ominoso movimiento telúrico se presentan en la quinta parte de este seminario denominada "Impacto de los sismos de 1985".

Siendo la ciudad de México mayor, demográficamente hablando, que más de la mitad de los países de la Tierra, es imprescindible que cuente con un aparato planificador que estructure un conjunto de planes y programas que regulen y gobiernen su dinámica expansión. En una sexta parte de este acto se presentará la evolución del proceso planificador de la capital del país y los planes vigentes que intentan normar su crecimiento.

La séptima y última parte de este seminario sobre la ciudad de México, presenta una imagen estimada de los aspectos demográfico-ecológicos de la ciudad de México hacia el año 2010. Junto con las investigaciones económicas, los ejercicios prospectivos de la capital del país son quizás sus aspectos menos estudiados. Ambos requerirán impulsarse significativamente para comprender los determinantes de su crecimiento y estar en posibilidad de realizar una programación realista de su evolución futura.

Las ponencias presentadas constituyen, sin lugar a dudas, una muy significativa colección monográfica de los aspectos más relevantes que conforman la fisonomía de la capital del país. Cabe anunciar que los documentos constituyen la parte analítica que se utilizará para la edición de un Atlas de la ciudad de México. Adicionalmente al material que en esta oportunidad se prescnta, está planteado un capítulo de los aspectos geográficos, otro de los históricos, y un último que contiene un conjunto de monografías sobre cada una de las 16 delegaciones y los 17 municipios conurbados del estado de México. El Atlas será coeditado por el Departamento del Distrito Federal y El Colegio de México. 


\section{TERCERA REUNIÓN NACIONAL SOBRE LA INVESTIGACIÓN DEMOGRÁFICA EN MÉXICO (3-6 DE NOVIEMBRE de 1986)}

Con una numerosa asistencia de demógrafos y otros profesionales interesados en los problemas de población se llevó a cabo del 3 al 6 de noviembre pasado la Tercera Reunión Nacional sobre Investigación Demográfica en México, organizada por la Sociedad Mexicana de Demografía (Somede) en las instalaciones de El Colegio de México.

Los antecedentes de esta reunión fueron las reuniones nacionales organizadas por el Consejo Nacional de Ciencia y Tecnología (Conacyt). La primera se efectuó en junio de 1977 y la segunda en noviembre de 1980.

Estas reuniones se llevaron a cabo dentro de las actividades del Programa Nacional Indicativo de Investigación Demográfica del Conacyt.

La Somede, que tiene como objetivo primordial contribuir al avance en los estudios de población, se propuso organizar la tercera reunión para continuar con las organizadas por el Conacyt (ya que el Programa Nacional Indicativo fue suspendido en 1982), y así mantener el interés en estas reuniones, que son el foro más relevante de los propios demógrafos.

Con la organización de esta tercera reunión, la Somede buscó recoger la experiencia acumulada de los demógrafos, científicos sociales y de otras áreas, tanto nacionales como extranjeras, interesados en el estudio de la población de México y su relación con otros factores, así como de aquellos involucrados en el desarrollo e implantación de políticas que a partir de un factor demográfico buscan mejoras en las condiciones de vida en el país.

El programa de la reunión estuvo integrado por 13 temas, obteniéndose un total de 116 ponencias registradas, además de los trabajos espontáneos que se presentaron fuera de programa. Las mesas de trabajo trataron temas tan interesantes como la crisis; la población y el desarrollo; mortalidad; comportamiento reproductivo; migración interna e internacional; trabajo; familia; envejecimiento de la población; salud; demografía histórica; reflexiones sobre la generación de información demográfica, requerimientos futuros de la investigación y docencia, y las perspectivas de la población para el siglo XXI.

En las ponencias presentadas (enriquecidas además por las discusiones tenidas en las distintas mesas), se mostraron los avances en el conocimiento de la dinámica demográfica, no sólo vista a través del análisis de sus componentes, sino de la explicación de los fenómenos que la modifican; esto gracias a los estudios interdisciplinarios y sobre todo a aquellos que están ubicados dentro de un contexto histórico y referidos a regiones particulares.

Tratando de obtener la máxima participación de los investigadores ubicados fuera del Distrito Federal, la Somede logró la asistencia y presentación de trabajos de investigadores provenientes de 19 entidades fede- 
rativas y de investigadores radicados en el extranjero.

Para la realización de la tercera reunión, la Somede contó con el apoyo de recursos financieros proporcionados por el Conacyt, el Consejo Nacional de Población, El Colegio de México, el Instituto Nacional de Estadística, Geografía e Informática, el Fondo de Naciones Unidas para Actividades en Materia de Población, la Universidad Nacional Autónoma de México, la Dirección General de Investigación Científica y Superación Académica de la SEP y la Dirección de Estudios de Población y Servicios de Planeación Familiar de la Secretaría de Salud.

La Somede revisa actualmente los trabajos presentados, con el objeto de poder ofrecer a la comunidad interesada en los estudios de población una selección que refleje de manera significativa los avances logrados en las investigaciones, las aportaciones metodológicas y aquellas temáticas de relevancia reciente para el conocimiento de la población mexicana.

Mercedes Pedrero Nieto Presidente de la Somede 
…... 